qui est en contradiction avec (12). Pour terminer la démonstration il suffit de prendre l'intégrale de la fonction $\Phi(x)$

$$
\int_{E_{f}} \Phi(x) d \mu=\gamma=\iint_{E E}|x-y|^{\alpha} d \mu d \mu+\int_{E} f(x) d \mu .
$$

\title{
Travaux cités
}

[1] F. Leja, O rozwartości arytmetycznej, geometrycznej $i$ harmonicznej zbioru, Zeszyty Naukowe UJ, Nr 3 (1957), p. 49-60.

[2] C. de la Vallée Poussin, Extension de la méthode du balayage de Poincaré et problème de Diriohlet, Ann. de l'Inst. H. Poincaré 2 (1932), Note I, p. 223.

[3] G. B jörck, Distributions of positive mass, which maximize a certain generalized, energy integral, Arkiv för Matematik, b. 3, 3 (1956), p. 255-269.

\section{Reģu par la Rédaction le 26.6.1960}

\section{Series containing the Hurwitz function}

by O. M. Fomenko (Krasnodar, USSR)

Notations. We shall denote by $\vartheta(s, a)$ the Hurwitz function which for $\sigma>1$, where $\sigma=\operatorname{Re} s$, is defined by the series

$$
\vartheta(s, a)=\sum_{n=0}^{\infty} \frac{1}{(n+a)^{s}} \quad(0<a \leqslant 1) .
$$

The Lerch function is defined by the series

$$
\varphi(x, b, s)=\sum_{n=0}^{\infty} \frac{e^{2 \pi i x n}}{(n+b)^{8}},
$$

where $\sigma>1$, and $b$ is a real constant different from 0 or a negative integer. Further, let $\omega$ denote a fixed value equal to 1 or -1 , and let

$$
\begin{aligned}
\vartheta(s, a, i) & =\sum_{n=0}^{i} \frac{1}{(n+a)^{s}} \quad(i=0,1,2, \ldots), \\
\vartheta(s, a,-1) & \equiv 0 .
\end{aligned}
$$

In the present note we shall deal with two series which contain the Hurwitz function: the formula

$$
\begin{aligned}
& \vartheta(s, a)-\vartheta(s, a, i)-\frac{1}{\left(a-\frac{1}{2}(\omega-1)+i\right)^{s-1}(s-1)} \\
& =-\sum_{j=0}^{\infty} \omega^{j+1} \frac{s(s+1) \ldots(s+j)}{(j+2) !}\{\vartheta(s+j+1, a)-\vartheta(s+j+1, a, i)\} \\
& \quad(i=0,1,2, \ldots)
\end{aligned}
$$

holds for all values of $s$. The proof of this formula consists of considering two particular cases $\omega=1$ and $\omega=-1$. Let for instance $\omega=1$. We shall rewrite the right-hand side of (3) in the following way: 
$-\frac{1}{s-1} \sum_{n=i+1}^{\infty} \frac{1}{(n+a)^{s-1}}\left\{\frac{(s-1) s}{2 !} \cdot \frac{1}{(n+a)^{2}}+\frac{(s-1) s(s+1)}{3 !} \cdot \frac{1}{(n+a)^{3}}+\ldots\right\}$

$=-\frac{1}{s-1} \sum_{n=i+1}^{\infty} \frac{1}{(n+a)^{s-1}}\left\{\left(1-\frac{1}{n+a}\right)^{1-s}-1-\frac{s-1}{n+a}\right\}$

$=-\frac{1}{s-1} \sum_{n=i+1}^{\infty}\left\{\frac{1}{(n+a-1)^{s-1}}-\frac{1}{(n+a)^{s-1}}-\frac{s-1}{(n+a)^{s}}\right\}$

$=\vartheta(s, a)-\vartheta(s, a, i)-\frac{1}{(a+i)^{s-1}(s-1)}$.

Sur les domaines de transitivité d'un groupe. de transformations

Changing the order of summation is correct because $\sigma>0$ and becanse of the absolute convergence of the series

$$
\begin{aligned}
& \sum_{n=i+1}^{\infty} \frac{1}{(n+a)^{\sigma-1}} \sum_{k=0}^{\infty} \frac{|s|(|s|+1) \ldots(|s|+k)}{(k+1) !} \cdot \frac{1}{(n+a)^{k+2}} \\
&=\sum_{n=i+1}^{\infty} \frac{1}{(n+a)^{\sigma}}\left\{\left(1-\frac{1}{n+a}\right)^{-|s|}-1\right\} .
\end{aligned}
$$

Since the series in the right-hand side of (3) is convergent for all values of $s$, the formula is proved by the method of analytic extiensions. For $\omega=1, i=0, a=1$ we obtain from (1) the Landau formula (see [1]). Formula (3) may serve for an analytic extension of Hurwitz function to the whole complex plane.

Let us consider the second series (which is also convergent for all values of $s$ ):

(4) $\quad p\left(\frac{1}{2}, 2 a+2 i+\frac{1}{2}(3-\omega), s\right)$

$$
\begin{array}{r}
=\sum_{j=0}^{\infty} \omega^{j} \frac{s(s+1) \ldots(s+j)}{(j+1) ! 2^{s+j+1}}\{\vartheta(s+j+1, a)-\vartheta(s+j+1, a, i)\} \\
(i=-1,0,1, \ldots) .
\end{array}
$$

The proof of (4) is analogous to the proof of (3), and we shall omit it.

For $\omega=1, i=-1, a=1$ we obtain the formula of Ramasvami (see [1]).

\section{Relerence}

[1] E. C. Titchmarsh, The Theory of the Riemann Zeta-function, Oxford 1951.

Reģu par la Rédaction le 31.5.1960 faltigleiten (Theorie der Transformationsgruppen $I, \mathrm{p}$. 224). 